\title{
L'ANAL YSE DES FIGURES PARENTALES DANS (LA PLACE) D'ANNIE ERNAUX (ETUDE ANALYTIQUE )
}

\section{Dr: Mohamed Ahmed Ibrahim El hanafy}

Maître de langue à la faculté des langues et de traduction

\section{1- Introduction :}

Le personnage ${ }^{1}$ du roman comme celui du cinéma ou celui du théâtre est indissociable de l'univers fictif auquel il appartient. Souvent, il est considéré comme le motif principal qui pousse l'écrivain à écrire son roman comme l'univers de Naguib Mahfouz qui détermine d'abord les personnages, ensuite, il commence à créer les événements.

" Le personnage se déploie dans l'univers romanesque; il vit des événements, il accomplit des actions, il parle, il discute, il pense, il réfléchit, il rêve, il aime, il hait, il entre en contact avec d'autres personnages, il se déplace, il habite des lieux qui le réconfortent ou le provoquent." ${ }^{2}$

Donc, Le personnage est le centre de la vie dans le roman, c'est la pierre de base autour de laquelle, tous les événements d'un roman tournent.

Selon Alain Robbe-Grillet, chaque personnage incarne un "type humain"3 seul caractère (le jaloux, le timide, l'avare,....)

La création d'un personnage avait donc autant d'importance que l'action et le style. Les critiques traditionnels voient que le vrai romancier est celui qui crée des personnages inoubliables

1 - Le mot "personnage" apparait en français au XIIIe siècle, mais l'acception de "personnage qui figure dans un ouvrage narratif", attestée pour la première fois en 1754 dans Quelques réflexions sur les Lettres persanes de Montesquieu, est relativement récente.

2 - Paquin (Michel): Lecture du roman, $\mathrm{La}$ lignée, 1984, p. 125

3 - Robbe Grillet (Alain): pour un nouveau roman, Paris, Gallimard,1963, p.33
"Les personnages ont un rôle essentiel dans l'organisation des histoires. Ils déterminent ces actions, les subissent, les relient et leur donnent sens." 4

Le personnage des parents occupent une place très considérable dans la plupart de romans d'Annie Ernaux. ${ }^{5}$

4 - Reuter : (Yves) Introduction à l'analyse $d u$ roman, Bordas, Paris, 1991 p.50.

5 - Selon Georges Décoté dans sa collection, Annie Ernaux est née le 1er septembre 1940 à Lillebonne. Elle passe son enfance en Normandie, à Yvetot. Elle appartient à un milieu social plutôt modeste, puisque ses parents sont ouvriers avant de devenir commerçants. Ernaux est une bonne élève, ce qui lui promet une réussite sociale plus élevée. Elle se rend régulièrement à l'école et montre beaucoup de facilités d'apprentissage. Ses parents la soutiennent, puisqu'ils la placeront dans une école privée pour qu'elle fasse de bonnes études. Par la suite, elle étudie à l'université de Rouen. Ses années d'étude montrent à Annie Ernaux à quel point les différences sociales sont importantes entre l'école où elle étudie et le milieu familial dont elle est issue. Ernaux exerce d'abord en tant qu'institutrice. En 1964, elle se marie et a son premier enfant. En 1967, elle obtient son Capes. En 1968 naît son second enfant. Dans les années 1970, elle enseigne notamment à Annecy, dans le collège d'Elvire. En 1971, Annie Ernaux est agrégée de lettres modernes.

En 1984, son ouvrage à caractère autobiographique, La Place, lui vaut le prix Renaudot.

Rapidement, Annie Ernaux décide de délaisser la fiction pure pour s'intéresser à la question de l'autobiographie. Elle se sert pour cela des souvenirs de sa propre enfance, dans l'épicerie et le café de ses parents, en Normandie : "Ils ont fait leur trou peu à peu, liés à la misère et à peine au-dessus d'elle. Le crédit leur attachait les familles nombreuses 
La Place est considéré comme un texte autobiographique, avec ce roman Ernaux a obtenu le Prix Renaudot, ce travail a une particularité car il n'a pas de chapitre, il dépend de la chronologie de vie de la narratrice et ses parents surtout la figure paternelle.

Dans "La place", Annie Ernaux raconte l'histoire de ses parents, leur ascension sociale, leurs conditions de vie et de travail, leurs espoirs...etc.

"Annie Ernaux ne cache pas la nature ouvertement autobiographique des récits où des preuves matérielles- photos, évocation de lieux réels, de faits précisviennent confirmer que les parents dont il est questions sont bien ceux de l'auteure, Annie Ernaux"6

Elle a essayé de transformer sa vie privée en production littéraire:

" Mes livres répondent, certes, au désir personnel que j'avais de faire entrer mes parents dans la littérature. Mais avec eux, c'est aussi toute une classe sociale que j'emmène." 7

\section{2- La problématique:}

Notre problématique consiste à analyser deux questions:

- Les figures parentales dans $\boldsymbol{L a}$ Place où nous allons traiter la description des personnages à

ouvrières, les plus démunies. Vivant sur le besoin des autres, mais avec compréhension, refusant rarement de I"marquer sur le comptel". (La Place) Son écriture mêle donc plusieurs dimensions : l'expérience individuelle et historique, le dépouillement $\mathrm{du}$ style, l'examen de la question sociale, qu'elle fait surtout au regard du trajet personnel de sa famille. Georges Décote, Le $21^{\text {ème }}$ siècle après 1945 , tombe 2 , p. 413

6 - Hugueny (Elise): Transgressions textuelles, déplacements littéraires et enjeux sociopolitique $d u$ transpersonnel dans l'œuvre d'Annie Ernaux, thèse de doctorat, université de Durham, Angleterre, 2007, p.73

7 - Entretien avec Jean Royer, Le Devoir, 26 mars 1988 travers trois aspects (l'aspect physique, l'aspect social et l'aspect psychologique et moral où nous avons amalgamé entre ces deux derniers aspects parce que l'un complète l'autre)

- La technique narrative qui va nous montrer comment l'auteure a participé comme un personnage dans les évènements du roman.

\section{3- La méthode suivie:}

Pour analyser les personnages, nous allons adopter une méthode mixte ou bien une méthode perspective éclectique qui amalgame entre les méthodes descriptive, sociologique.

\section{4- Corpus de l'étude:}

On va appliquer cette méthode sur

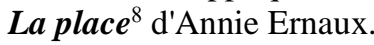

\section{5-Originalité du corpus:}

Avant de commencer notre analyse, nous avons un problème concernant la classification de notre roman "La place":

(Est-ce que c'est un roman autobiographique ou autofictionnel?)

Ce problème nous attire à distinguer entre les deux genres, nous pouvons dire que les événements du roman autobiographique tournent autour de l'écrivain où il raconte sa vie d'une façon réelle, mais quand l'écrivain mélange entre la réalité et l'imagination, dans ce cas, l'écrivain écrit un roman autofictionnel.

"La présence du genre autobiographique ne s'accroit qu' à partir d'un souci philosophique moderne, qui pousse l'homme à se pencher sur lui-même et sur son passé."

En réalité, le néologisme "autofiction" a été créé par Serge Dobrovsky en 1977, mais il y a d'autres

8 - Ernaux (Marie): La place, Gallimard, Paris, 1996.

9 - Jopeck (Sylvie): La photographie et l'autobiographie, anthologie, Paris, Editions Gallimard, 2004, p.7 
écrivains qui le nomment par une autre manière ${ }^{10}$.

Quant à 'Annie Ernaux, elle mélange entre la mémoire et l'imagination, mais, elle s'intéresse au premier rang à la mémoire. Selon Annie Ernaux: "La mémoire individuelle fixe visuellement le souvenir de détails, de paroles ou de sentiments"11

Ernaux a tenu à souligner tout au long de son parcours, la dimension sociale de son travail, soutenant que sa vie personnelle, en tant que thème central de son écriture, l'intéresse uniquement dans la mesure où elle est connectée à celle des autres, où elle est un reflet de celle des autres où elle dit:

" Je me considère très peu comme un être unique, au sens d'absolument singulier, mais comme une somme d'expériences, de déterminations aussi, sociales, historiques, sexuelles, de langages, et continuellement en dialogue avec le monde (passé et présent), le tout formant, oui, forcément, une subjectivité pour retrouver, dévoiler des mécanismes ou des phénomènes plus généraux, collectifs" 12

10 - "surfiction" (Raymond Federman), "nouvelle autobiographique" (Alain Robbe Grillet), "fiction autobiographique postcoloniale" (Rachid Boudjedra), "autofiction biographique" (Vincent Colonna), "autobiographie génétiquement modifiée" (Philippe Vilain), "autophotobiographie" (Sophie Calle), "autonarration" (Schmitt) ${ }^{10}$ ?, Annie Ernaux refuse toutes ces nominations en suggérant un autre nom "auto-socio-biographie", un genre d'écriture où "toute fictionnalisation des événements est écarté."

Romeral (Francuscaà: Les Années 80 et l'épanouissement de l'autofiction. Intercambio, $2^{\text {ème }}$ série, vol, 6, 2013, p. 147

11 - Ernaux (Annie): L'écriture comme un couteau, entretien avec Fréderic, Yves Jeannet, Paris, Stock, 2003,p.41

12 - Ernaux (Annie): L'écriture comme un couteau, entretien avec Fréderic,p.: 153
Outre, nous pouvons dire que la Place représente un nouveau genre appelé "une nouveau-socio-biographie" nous soutenons l'avis de la chercheuse iranienne Layla Ghafouri Gharavi:

"La Place est un récit novateur marqué par une écriture atypique et épurée. La Place constitue un tournant dans la pratique de l'écriture de l'auteur: elle ne se présente plus comme le personnage central du livre mais dans plusieurs passages, c'est son père qui domine le récit:"13

Donc, nous pouvons classifier $\mathrm{La}$ Place comme une œuvre auto-sociobiographie, l'auteure elle-même estime beaucoup ce travail et le considère comme un point de transformation en ce qui concerne sa vie littéraire:

"La Place est un livre qui a orienté différemment mon écriture et m'a fait poser un ensemble de questions: que puisje faire par rapport à cette vie qui n'est plus? Que puis-je faire de vrai? Quelle est ma place dans le texte? Dans le champ littéraire? Par rapport au lecteur?"14

Enfin, il faut signaler que plusieurs écrivains ont beaucoup de problèmes dans leur vie privée ou sociale, pour cela, ils y trouvent la solution dans la fiction.

Selon Stendhal: "Il faut pour les arts des gens un peu mélancoliques et assez malheureux." 15

\section{6- Analyse du corpus:}

Nous pouvons d'abord signaler que la caractérisation du personnage peut être explicite ou implicite. L'écrivain a recours à la caractérisation explicite pour présenter l'aspect physique et sociale, dans ce cas,

13 - Ghafouri (Layla): La Revue de Téhéran, mensuel culturel iranien en langue française, n. 94 , septembre, 2013, p. 2

14 -Ernaux (Annie) "écrire, pourquoi?" Entretien avec Raphaëlle Rerolle, Editions de la bibliothèque publique d'informationCentre Pompidou, 2010, p. 4

15 - Rohou (Jean): Les études littératures "guide de l'étudiant", Nathan, Tours, 1993, p. 34 
l'écrivain s'intéresse à l'allure extérieure du personnage lui-même et ses relations avec les autres, mais, la caractérisation est implicite quand l'écrivain plonge dans les fonds de ses personnages pour nous présenter l'aspect moral ou bien l'aspect psychologique.

"Les données qui forment le signalement du personnage sont le fruit de procédés de caractérisation, c'est -à-dire de techniques utilisées par le romancier pour conférer à cet être de papier et de mots les caractéristiques, les attributs des personnes."16

Nous allons analyser le personnage du père car il est le personnage principal de notre roman mais en même temps, nous ne pouvons jamais négliger le personnage de la mère qui a joué le rôle essentiel dans la vie de son mari.

Avant de traiter les figures parentales, Il faut jeter la lumière sur les grands-parents de la narratrice (Annie Ernaux) pour mettre en relief leur influence très apparente sur les parents d'Annie Ernaux.

" La narratrice raconte la vie de ses grands-parents afin de donner un cadre à l'histoire familiale: le retour aux origines est un passage quasi obligé, presque un topos de la littérature autobiographique. L'histoire des grands-parents permet de mieux comprendre d'où vient le narratrice, comment ses parents sont devenus ce qu'ils sont et comment ils lui ont permis d'être qui elle est. ${ }^{\prime 17}$

La narratrice nous a parlé de son grand-père car il a laissé une grande influence sur la conduite et la manière de vie de son père pour cela, la narratrice met l'accent sur les vices et les défauts de son grand-père où elle s'intéresse seulement à

\footnotetext{
16 - Rohou (Jean): Les études littératures "guide de l'étudiant", p. 71

17 - Blévennec (Emile): La place d'Annie Ernaux, formes du récit aux $20^{\text {ème }}$ et $21^{\text {ème }}$ siecle, Edition d'Emile Péron Blévennec, p. 3
}

l'aspect moral elle croit que son père est considéré comme l'ombre de son père (le grand- père de la narratrice):

"Mon grand-père travaillait donc dans une ferme comme charretier. L'été, il faisait aussi les foin, la moisson. Le samedi soir, il rapportait à sa femme toute sa paye et elle lui donnait son dimanche pour qu'il aille jouer aux dominos, boire son petit verre. Il rentrait saoul, encore plus sombre. Pour un rien, il distribuait des coups de casquette aux enfants. C'était un homme dur, personne n'osait lui chercher des noises. Sa femme ne riait pas tous les jours, Cette méchanceté était son ressort vital, sa force pour résister à la misère et croire qu'il était un homme. Ce qui le rendait violent, surtout, c'était de voir chez lui quelqu'un de la famille plongé dans un livre ou un journal. Il n'avait pas eu le temps d'apprendre à lire et à écrire. Compter, il savait" 18

L'autre personnage qui complète le cadre familial de la narratrice est la grandmère. Nous remarquons que l'auteure cite quelques aspects éclairés ou bien les avantages de sa grand-mère où Ernaux sympathise avec elle à cause de son amour pour sa mère. La narratrice concentre pour nous présenter sa grand-mère comme une femme plus civilisée que son grand-père.

"Ma grand-mère avait même de la distinction, aux fêtes elle portait un faux cul en carton et elle ne pissait pas debout sous ses jupes comme la plupart des femmes de la campagne, par commodité"19 A travers cette citation, la narratrice veut dire que sa grand-mère est une femme propre elle s'intéresse à sa propriété et ne fait pas comme les autres femmes de la campagne

" Ma grand-mère, elle, avait appris à l'école des sæurs". ${ }^{20}$

18 - Ernaux (Marie): La place, Gallimard, Paris, 1996, p. 25

19 - La place : p. 19

20 - La place: p. 26 
"Comme les autres femmes du village, elle tissait chez elle pour le compte d'une fabrique de Rouan. "21

Ces deux citations nous montrent que cette grand-mère fréquente l'école, elle est une femme cultivée, après être rentrée à la maison, elle commence à tisser pour le compte d'une fabrique car elle est une femme coopérative. Bref, la grand-mère est une femme cultivée, active, coopérative.

A travers la présentation de la vie de grands-parents, le lecteur peut former une idée globale sur les conditions de vie dans les campagnes françaises à la fin du $19^{\text {ème }}$ siècle et le $20^{\text {ème }}$ siècle. En effet, leur vie n'était pas aisée; les enfants étaient mis au travail dès leur plus jeune âge, comme le grand-père de la narratrice qui a commencé à travailler à l'âge de huit ans sans apprendre à lire ni à écrire à l'école. Les seuls moments de réjouissance étaient les noces et les communions, occasions pour tout le village de manger à outrance. La religion joue un rôle très important dans la vie des paysans:

"Le signe de croix sur le pain, la messe, les pâques. Comme la propreté, la religion leur donnait la dignité, ils habillaient en dimanche." 22

\subsection{Le père:}

6.1.1. L'aspect physique:

"La description de l'aspect physique général du personnage est, parmi les façons de caractériser un personnage, l'une des plus employées. Le narrateur précisera les traits propres à son visage, décrira ses vêtements, soulignera ses tics, caractérisera sa voix, bref, il rendra son aspect physique général." 23

Il faut signaler que le père est la figure centrale du roman autour laquelle se fixe toute la narration. La narratrice commence

\footnotetext{
${ }^{21}$ - La place: p.p. 26,27

22 - La place.: p. 28

23 - Paquin (Michel): Lecture du roman,op.cit. p. 72
}

la description physique de son père après sa mort:

La narratrice nous décrit le visage du père après sa mort les yeux, les lèvres, les gencives, le nez.....etc. Elle utilise le mot" figure" pour indiquer qu'il est mort, elle le décrit comme un statue pas un homme, elle manque les sentiments de tristesse. Cette méthode de description montre la nature de la relation qui se trouve entre la narratrice et son père.

"Je revois seulement les yeux de mon père fixant quelque chose derrière moi, loin, et ses lèvres retroussées au-dessus des gencives. Je crois avoir demandé à ma mère de lui fermer les yeux. "24

"En quelques heures, la figure de mon père est devenue méconnaissable. Le nez avait pris toute la place dans la figure creusée. ${ }^{\prime 25}$

Ernaux nous présente une image rhétorique très expressive où elle compare l'odeur très mauvaise du corps de son père par l'odeur de fleurs oubliées dans un vase d'eau croupie. Outre, elle utilise la chosification pour parler le corps de son père quand l'on l'a mis dans un sac de plastique.

"L'odeur est arrivée le lundi. Je ne l'avais pas imaginée. Relent doux puis terrible de fleurs oubliées dans un vase d'eau croupie" 26

"Le corps a dî être enveloppé dans un sac de plastique et trainé, plus que transporté, sur les marches, jusqu'au cercueil posé au milieu du café fermé pour une heure." 27

A travers ces passages courts, la narratrice nous présente une description méprisée pour le corps de ce pauvre père. Cette présentation nous montre la proportion de la distance entre la fille (la narratrice) et son père car le lecteur ne voit que aucun singe qui montre la tristesse

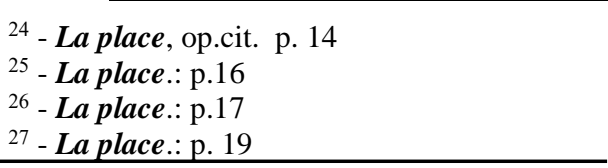


d'une fille qui a perdu son père, donc dès le début du roman on peut découvrir la forme de la relation entre la narratrice et son père. Dans les passages suivants, elle complète sa description physique qui montre la vie misère de son père.

"Pour manger, il ne se servait que de son couteau Opinel. Il coupait le pain en petits cubes, déposés près de son assiette pour y piquer des bouts de fromage, de charcuterie, et saucer. Me voir laisser de la nourriture dans l'assiette lui faisait deuil. On aurait pu ranger la sienne sans la laver. L e repas fini, il essuyait son couteau contre son bleu. S'il avait mangé $d u$ hareng, il l'enfouissait dans la terre pour lui enlever l'odeur." 28

"Il dormait toujours avec sa chemise et son tricot de corps. Dans la cour, l'hiver, il crachait et il éternuait avec plaisir." 29

Toutes les citations précédentes mettent en relief la conduite et les sentiments de la narratrice envers son père où elle le critique sévèrement, il agit grossièrement, il n'est pas propre et très avare en même temps.

Le portrait du père s'achève avec la description de son agonie et de sa mort, le livre se terminant comme il avait commencé. C'est aussi l'occasion pour l'auteur de dresser un bilan de la relation entretenue avec son père.

"Mon père respirait difficilement, les yeux grands ouverts. J'ai entendu ma mère marcher lentement au-dessus, commencer à descendre. J'ai cru, malgré son pas lent, inhabituel, qu'elle venait boire son café. Juste au tournant de l'escalier, elle a dit doucement: "C'est fini." 30

\subsubsection{L'aspect social:}

L'individu appartient à un groupe social, le personnage reflète un milieu (par ses vêtements, son langage, son idéologie). Il faut signaler que le langage

\footnotetext{
28 - La place.: p.68

29 - La place.: p.69

30_ La place, p. 110
}

$\mathrm{du}$ personnage constitue un moyen privilégie de caractérisation, il utilise un style soutenu ou populaire, d'autre part, la langue peut aussi bien constituer un révélateur qu'un masque. Certains indices parfois permettent de déceler un éventuel décalage entre le langage qu'utilise un personnage et ce qu'il est "réellement".

Le passé familial du personnage a une fonction psychologique: il permet souvent d'expliquer certains problèmes actuels par le recours à un problème relationnel passé, tel, par exemple, le manque d'amour paternel. Le personnage entretient de nombreux rapports avec la société, lesquels permettent de dévoiler sa personnalité en fixant son rôle social, sa position.

L'œuvre d'Annie Ernaux est très fortement marquée par une démarche sociologique.

"Ainsi, ses ouvrages traitent du métissage social de sa trajectoire (fille de petits commerçants devenue étudiante, professeure puis écrivain) et des mécanismes sociologiques qui l'accompagnent" 31

Nous pouvons dire que les relations entre le père et sa fille sont conflictuelles car leur vue pour la vie est totalement différente où le père voit le travail est le travail manuel, les études ne servent qu'à obtenir une bonne situation dans la société. Cette différence entre le père et sa fille participe à élargir la distance entre les deux.

La narratrice a réussi à dessiner une image qui reflète les conditions très difficiles dans lesquelles son père vivait en montrant qu'il n'avait pas la volonté de changer sa vie. A l'autre côté, Annie Ernaux veut changer la place " à double sens" la place comme une classe sociale et la place comme un lieu, elle voit sa région Normandie basse et lourde, cette région

31 - Hugueny (Elise): op.cit. p. 74 
parait comme un couvercle tassé, étroite et empesée.

"Ils habitaient une maison basse, au toit de chaume, au sol en terre battue. Il suffit d'arroser avant de balayer. Ils vivaient des produits $d u$ jardin et du poulailler, du beurre et de la crème que le fermier cédait à mon grand-père." 32

Pour la vie scolaire de son père, Ernaux nous offre une image très misérable et sombre pour cette vie qui le pousse à laisser l'école pour travailler avec son père dans les champs et les fermes.

" Il faisait deux kilomètres à pied pour atteindre l'école. Mon père manquait la classe, à cause des pommes à ramasser, $d u$ foin, de la paille à botteler, de tout ce qui se sème et se récolte. Quand il revenait à l'école, avec son frère ainé, le maitre hurlait "Vos parents veulent donc que vous soyez misérables comme eux." 33

" Il couchait au-dessus de l'étable, une paillasse sans draps. Il est resté gars de ferme jusqu'au régiment" ${ }^{\prime \prime 3}$

" Des vaches du matin à celles du soir. Mon père travaillait la terre des autres, il n'en a pas vu la beauté" 35

En ce qui concerne sa communication avec les autres, c'est un personnage ignoré, peu cultivé, pas civilisé, il ne sait pas traiter avec les nouvelles inventions comme la machine à laver, la télévision, il est seulement un paysan très simple et naïf.

" Bavard au café, en famille, devant les gens qui parlaient bien." 36

" Faire paysan signifie qu'on n'est pas évolué, toujours en retard sur ce qui se fait, en vêtements, langage, allure. Il s'assoit devant la machine à laver qui tourne, et reste là, pensif, à fixer le linge brassé derrière le hublot. A la fin, il se

32 - La place: p. 27

33 - La place.:.p.29

34 - La place.: p. 31

35 - La place.: p. 33

36 - La place.: p.63 lève, hoche la tête et dit à sa belle-fille: On dira ce qu'on voudra, la télévision c'est pas au point." 37

La narratrice voit que l'enseignement est le moyen efficace pour changer cette médiocre position. La fille essaie de fuir de cette misère et de cette classe sociale ou bien de "cette place sociale," ${ }^{38}$ elle espère améliorer sa vie et vivre dans un autre milieu, pour cela, elle déploie beaucoup d'efforts pour réaliser ce but et avoir un poste qui lui garantit une vie de large.

"L'espérance que je serais mieux que lui." 39

" Il s'énervait de me voir à la longueur de journée dans les livres.

Les études n'avaient pas pour lui de rapport avec la vie

ordinaire." 40

" On ne savait pas se parler entre nous autrement que d'une manière râleuse. Le ton poli réservé aux étrangers. Il n'avait pas appris à me gronder en distingué."41

" La dispute éclatait à table pour un rien. Je croyais toujours avoir raison parce qu'il ne savait pas discuter. ${ }^{42}$

Le père s'énerve toujours quand il voit sa fille lit les livres ou étudie quelque chose, la narratrice voit que son père essaie de l'attirer à sa classe (la classe des paysans et des ouvriers mais, elle veut être mieux que son père qui n'a pas le mécanismes de la discussion avec sa fille, pour cela la dispute éclate toujours dans les coin de la maison.

En fait, L'école représente la pierre angulaire dont la narratrice dépend.

37 - La place.: p.70

38 - Quelques lecteurs peuvent comprendre faussement le titre du roman, "la place" ne signifie pas la place spatiale mais c'est la classe sociale.

39 - La place: p.74

40 - La place.: p.p. 81,82

41 - La place.: p. 72

42 - La place: p. 83 


"Ainsi, l'école s'apparente
métaphoriquement à une autre ligne de vie possible que suit la jeune fille par intuition, comme appelée par le pressentiment qu'il lui faudra coute que coute combler les vides du silence paternel sur cet échec scolaire par défaut; défaut d'éduction bien sûr. Ce qu'elle n'a pu donner au père- la possibilité de se singulariser- l'école la donnera donc) la fille. Et le cri du maitre en colère contre les parents massacreurs d'éducation par nécessité ne cessera sans doute jamais de retentir en elle à la fois comme une offense à réparer et une supplique à écouter: "Vos parents veulent donc que vous soyez misérables comme eux!"'"43

Bref, toutes les citations précédées mettent en relief la grande différence entre la fille et son père où elle le critique toujours soit au niveau personnel, soit au niveau social. La relation entre la narratrice (la fille) et son père change à partir du moment où elle commence à grandir et découvrir le monde des livres et de la culture. Donc, nous pouvons confirmer que la critique continuelle de la narratrice (la fille) a participé à se creuser entre elle et son père une certaine distance qui se manifeste par l'incapacité à communiquer d'une part, et d'autre parce que la narratrice (Annie Ernaux) se considère comme une petite bourgeoise.

\subsubsection{L'aspect psychologique ${ }^{44}$ :}

43 - Soron (Antony): La Place d'Annie ou le délit d'appartenance normande, Sorbonne, Paris, p. 5

44 - L'expression "analyse psychologique" apparait dès le $18^{\text {ème }}$ siècle, notamment dans l'Essai sur l'origine des connaissances humaines (1746) de Condillac. Au $19^{\text {ème }}$ siècle, elle a été utilisé dans des sens divers par une série d'auteurs, entre autres, Maine de Biran, Taine, Laromiguière, Wundt.Pierre Janet utilisait cette expression dès la fin des années 1880, sans toutefois en faire un concept à lui, contrairement à ce que tentera
L'analyse psychologique est la base de toute œuvre qui respecte le modèle du roman traditionnel. La description y a pour but de dévoiler et de justifier la psychologie des personnages. Les actions sont expliquées.

"elles sont le plus souvent préparées narrativement par un psycho-récit ou un monologue intérieur, et suivies en général de bilans du même ordre." 45

La description psychologique est considérée comme "la science de l'âme" de la "vie mentale", du "comportement" ou encore comme l'ensemble des traits de caractère d'un individu ou d'un groupe.

La psychologique des personnages est, contrairement à ce qu'on pourrait croire, très importante, c'est elle qui fait qu'un personnage réagit de telle façon plutôt qu'une autre façon. Pour bien rendre les personnages, le narrateur précise, dans la plupart des cas, leurs grands traits psychologiques ou le texte s'attache à l'expression des sentiments, s'intéresse à leurs manifestations extérieures (larmes, sourires, gestes significatifs).

Le romancier prête aux personnages des pensées, des valeurs qui sont parfois les siennes. Cette caractérisation psychologique ou morale sera soit sommaire, soit détaillée.

Le psychologique français Pierre Janet l'exprime clairement:

"Le fait psychologique n'est ni spirituel, ni corporel; il se passe dans l'homme tout entier, puisqu'il n'est que la conduite de cet homme prise dans son ensemble." 46

D'autre part, L'analyse psychologique dépend d'une connaissance approfondie de la biographie et de la

de faire Freud pour sa version allemande "Psychanalyse."

45 - Dugast-Portes (Francine), Le nouveau roman. Une césure dans l'histoire du récit, Paris, Nathan. 2001, p. 80

- BOURNEUF (Roland) : L'univers du ‘ Roman, Presses Universitaires de France, 1975 , p. 168 
personnalité. Nous préférons finir l'analyse du personnage par l'aspect psychologique parce que nous voyons que l'aspect psychologique est considéré comme le résultat naturel pour l'introduction qui comprend (l'aspect physique et social), sans doute l'allure physique et la classe sociale laisse ses influences très fortes et touchantes sur l'âme et la conduite du personnage.

Pour Freud, la création littérature comme le rêve "décharge psychique d'un désir en état de refoulement, mais il faut dire que l'étude analytique de l'ouvre littérature repère aussi des structures caractéristiques du rapport entre le désir et son objet et du conflit entre le désir et l'interdiction figurée par le père et la mère, l'état ou la loi, la raison, la conscience ou la morale......etc. ${ }^{47}$

Annie Ernaux a bien décrit physiquement et socialement son père mais elle n'a pas réussi à faire l'analyse psychologique pour lui parce que les mauvaises conditions de vie et la conduite très grossière de son père laissent des effets psychologiques sur Annie Ernaux elle-même, quand même, on peut dégager quelques signes qui traduit les l'état psychologique du père.

"Je voudrais dire, écrire au sujet de mon père, sa vie, et cette distance venue à l'adolescence entre lui et moi. Une distance de classe, mais particulière, qui n'a pas de nom. Comme de l'amour séparé. Par la suite, j'ai commencé un roman dont il était le personnage principal. Sensation de dégout au milieu du récit" 48

" Mon père était gai de caractère, joueur, toujours prêt à raconter des histoires, faire des farces. ${ }^{49}$

\footnotetext{
47 - Rohou (Jean): Les études litteratures "guide de l'étudiant", Nathan, Tours, 1993, p. 32

48 - La place. p. 23

49 - La place.: p. 32
}

" Il était sérieux, c'est-à-dire, pour un ouvrier, ni feignant, ni buveur, ni noceur"50

"Mon père est entré dans la catégorie des gens simples ou modestes ou braves gens $^{\prime 51}$

"A cette époque, il a commencé d'entrer dans des colères, rares; mais soulignées d'un rictus de haine." 52

Ces passages montrent clairement la personnalité très simple du père, il est satisfaisant, il n'a pas de complexes psychologiques, mais on peut dire que la distance qui se trouve entre la fille qui méprise sa classe sociale laisse des influences psychologiques sur la personnalité de la fille pas sur le père.

Nous remarquons qu'Annie Ernaux s'oppose à Freud et sa théorie (la complexe d'Electre), cette théorie qui montre que les filles aiment leurs pères plus que leurs mères, cette complexe ne parait pas évidemment dans notre récit car la fille est liée étroitement à sa mère.

Donc ce roman est considéré comme un exemplaire qui prouve l'échec de la théorie freudienne parce que l'enfant aime la personne qui le traite doucement soit le père soit la mère.

\subsection{Le mère:}

La deuxième figure parentale dans notre roman, c'est la mère qui complète l'action. La narratrice déclare que le roman raconte au premier rang la vie et la mort de son père et sa relation avec lui mais elle ne peut pas négliger le rôle très important et très axé de la mère.

\subsubsection{L'aspect physique:}

" Elle voulait copier la mode des journaux, s'était fait couper bien les cheveux parmi les premières, portait des robes courtes et se fardait les yeux; les ongles des mains. Elle riait fort. En réalité, jamais elle ne s'était laissé toucher

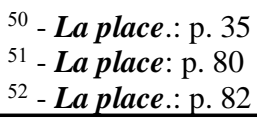


dans les toilettes. Tous les dimanches elle allait à la messe et elle avait ajouré ellemême ses draps, bordé son tricot. C'était une ouvrière vive, répondeuse. Une de ses phrases favorites: "je vaux bien ces genslà." 53

"Sur la photo du mariage, on lui voit les genoux. Elle fixe durement l'objectif sous le voile qui lui enserre le front jusqu'audessus des yeux. Elle ressemble à Sara Bernardt." 54

La narratrice ne s'est pas intéressée à la description physique de la mère parce que le personnage central dans le récit, c'est le père d'une part, et d'autre, Annie Ernaux essaie de mettre en relief le rôle social très important de la mère dans la vie du père.

\subsubsection{L'aspect social:}

La mère vient d'un milieu modeste mais elle est ambitieuse de changer sa position social en essayant de monter sur l'échelle social, pour cette raison son influence était très forte sur son mari, elle prend toujours le dessus sur lui.

" Elle était patronne, elle ne disait pas comme d'autres femmes "mon mari va me disputer si j'achète ça, si je vais là." Il lui laissait le soin des commandes et du chiffre d'affaires. C'était une femme qui pouvait aller partout, autrement dit, franchir les barrières sociales. "55

Cette différence d'aisance entre le père et la mère apparait également dans leur rapport à la langue. Le père refuse d'employer des mots "à la mode" alors que la mère est attentive et réceptive aux évolutions de son temps.

En effet, la différence entre la fille et sa mère est une différence entre le rêve et la réalité, la narratrice espère changer sa place sociale et son milieu, elle fait tous ses efforts pour réaliser ce rêve, mais sa mère n'éloigne pas de sa réalité, elle veut

53 - La place.: p. p.36,37

54 - La place.: p. 37

55 - La place: p. 43 vivre une vie plus facile ou large si c'est possible.

"le rapport de la fille à sa mère l'écart entre l'image sociale et la réalité vécue."

\subsubsection{L'aspect psychologique:}

En ce qui concerne l'aspect psychologique de la mère, nous voyons qu'elle a l'ambition d'améliorer les conditions de vivre de sa famille, pour cela, elle est le vrai responsable de la famille. C'est elle qui lance l'idée du commerce et permet ainsi à son mari de s'extraire du monde ouvrier où elle croit que la possession d'un petit commerce représente pour elle le moyen de s'élever au-dessus de sa condition d'ouvrière.

"Le père l'admire car elle est capable de "franchir les barrières sociales" 57

C'est une femme très solide, elle a pu diriger facilement le café et l'épicerie après la mort de son mari et séparer entre sa tristesse et son devoir.

" Ma mère est redevenue calme. Elle servait les clients comme avant. Seule, ses traits s'affaissaient. Chaque matin, tôt, avant l'ouverture du commerce, elle a pris l'habitude d'aller au cimetière"

Elle est fière de sa réussite soit dans la direction du commerce soit la direction de la maison. Nous pouvons dire la réussite de la mère et son ambition attire la fille envers elle parce qu'elles ont le même but (monter sur l'échelle pour changer leur position sociale envers la classe bourgeoise), cette raison nous explique la distance qui se trouve entre la fille et son père d'une part, et d'autre, elle indique le rapprochement qui se trouve entre la fille et sa mère.

\subsection{La technique narrative:}

Il est évident qu'un événement ne peut pas se raconter puisque le roman est une histoire racontée par quelqu'un.

56 - Vercier (Bruno): La littérature en France depuis 1968, Bordas, 1982, P.P. 240, 241;

57 - La place.: p. 43

58 - La place.: p. p. 22,23 
La personne qui lit un roman, réfléchit sur ce qui se passe, à travers l'écriture en essayant de répondre à la question «qui parle ?»

"(Qui parle?) dans le récit n'est pas qui écrit (dans la vie) et (qui écrit?) n'est pas qui est? "59

Donc, il ne faut pas mélanger entre l'écrivain et le narrateur, l'auteur écrit une œuvre pour un lecteur virtuel, dans la fiction, mais un narrateur raconte une histoire à un narrataire.

L'auteur est la personne réelle qui vit ou a vécu, dans un temps et dans un lieu déterminé. Il lance une pensée et peut faire l'objet d'une enquête biographique. Il inscrit généralement son nom sur la couverture du livre que nous lisons.

Donc, le narrateur est considéré comme l'élément le plus important en ce qui concerne la vision dans le roman ou bien dans la fiction, à travers l'exposition du narrateur, le lecteur peut comprendre les paroles et les faits des personnages

" Le narrateur contrôle nos impressions et dirige, par son ton, ses façons de parler, ses opinions et ses préjugés, notre interprétation $d u$ spectacle qui nous est présenté dans le récit, le narrateur sert donc d'intermédiaire entre l'auteur, qui a écrit le récit, et le lecteur, qui le lit..."60

Généralement, nous divisons le narrateur en trois types.

- Narrateur à la première
personne (je).
- Narrateur à la troisième
personne limitée (il).
- Narrateur à la première
personne omnisciente (il).

Notre narratrice utilise le premier et le deuxième type où elle utilise le pronom (je), cependant, le personnage principal dans "La place" est le père de la narratrice,

(1) Barthes (Roland) : Introduction à l'analyse structurale des récits, Seuil, 1977, p. 40

${ }_{60}$ - Preckshot (Judith): L'analyse du récit: lexique commenté, p. 3 donc nous voyons que "La place" amalgame entre l'autobiographie d'Annie Ernaux et la biographie de son père.

Elle utilise souvent des mots qui dévoilent clairement de sa personnalité comme "Mon père, ma mère, ma grand-mère, je,..." Mais quelquefois, elle a recours à la troisième personne comme "il, elle, ils, son, sa, ses ...etc."

Comme tout texte autobiographique, La place est un récit rétrospectif par laquelle la narratrice revient sur des événements de son passé. Tout au long du récit, la narratrice évoque son enfance et son adolescence et raconte ses souvenirs, Annie Ernaux comme une narratrice joue un rôle double où elle est un personnage dans le récit et la narratrice en même temps:

"Je travaillais mes cours, j'écoutais des disques, je lisais, toujours dans ma chambre, je n'en descendais que pour me mettre) table. On mangeait sans parler. Je ne riais jamais à la maison. Je faisais de "l'ironie" c'est le temps où tout ce qui me touche de près m'est étranger. J'émigre doucement vers le monde petitbourgeois." 61

Enfin, nous pouvons dire qu'Ernaux adore la dualité dans sa vie, peut-être parce qu'elle est l'unique fille de ses parents, elle mélange toujours entre la sociologie et la littérature, entre la narration extérieure et la narration intérieure.

\section{7- Conclusion:}

Pour conclure notre travail, on va résumer nos conséquences en quelques points:

Premièrement, nous pouvons classifier ce travail sous un nouveau genre littéraire, "une nouveau-socio-biographie" pour cela, ce roman est considéré comme une station très importante dans la procession littéraire française. Vraiment, la littérature est le produit de la société et du

61 - La place: p. 61 
milieu, Ernaux a bien réussi de lier entre la société et la littérature et fait de la littérature comme un miroir qui reflète les conditions de la vie humaine.

Deuxièmement, nous pouvons dire que Annie Ernaux a bien choisi le titre de son roman "La place", ce titre a une valeur symbolique, la place ici ne signifie pas un lieu mais c'est la place de la fille dans sa famille et sa société (la classe sociale), Annie Ernaux veut monter sur l'échelle sociale vers la bourgeoisie où elle a déclaré dans un programme télévisé qu'elle était déchirée entre le milieu bourgeois et le monde très pauvre de son père. La relation entre le père et la fille évolue lors du passage de l'enfance à l'adolescence. Enfance, la fille semble proche de son père et partage avec lui des moments simples, en entrant dans l'adolescence, la fille fait également son entrée dans le monde bourgeois. A partir de ce moment-là, elle commence à mépriser le milieu de son père et se dirige envers sa mère, donc, plus la fille grandit, plus les liens se resserrent entre elle et la mère.

Troisièmement, nous voyons que notre méthode appliquée convient la technique avec laquelle Annie Ernaux a construit ses figures parentales où elle a traité ses personnages à travers trois aspects (physique, social ou moral, psychologique), mais elle s'est intéressée plus à l'aspect social qui est considéré comme l'axe principal dans le roman parce qu'elle voulait changer sa position sociale.

Quatrièmement, ce roman prouve l'échec de la théorie freudienne en ce qui concerne le complexe d'Electre et Edipe car la fille méprise son père et son milieu, par contre, sa mère est considérée comme son exemplaire.

Cinquièmement, il faut noter que les descriptions et les citations de choses et des événements vus et entendus, grandissent la réalité et la sincérité au travail, c'est pourquoi, "La place" a réalisé un énorme succès parce qu'il raconte des événements réels, nous le considérons comme le chef d'œuvre d'Annie Ernaux avec lequel, elle a pu obtenir le prix Renaudot.

Finalement, au niveau de la technique romanesque, "La place" appartient au roman traditionnel car Annie Ernaux a appliqué les règles de la technique romanesque traditionnel (l'espace, le temps, les personnages, l'action, les thèmes), mais nous observons qu'elle a négligé de citer le nom de son village où elle habitait où elle donne toujours un symbole comme (Y), (I), certains croient que Annie Ernaux suit ici la même manière de nouveau roman qui ne s'intéresse pas à la citation de l'espace d'une façon traditionnelle mais on peut dire qu' elle veut montrer que le lieu où elle vit ne représente aucune importance aucune valeur pour elle.

\section{Bibliographie}

\section{I- Corpus:}

Ernaux (Marie): La place, Gallimard, Paris, 1996.

II- Références en francais:

1- BARTHES (Roland)
Introduction à l'analyse structurale des récits, Seuil, 1977

2- BLEVENNEC (Emile): La place d'Annie Ernaux, formes du récit au $20^{\text {ème }}$ et $21^{\text {ème }}$ siècle, Edition d'Emile Péron Blévennec.

3- BOURNEUF (Roland) : L'univers du Roman, Presses Universitaires de France, 1975

4- DUGAST-PORTES (Francine), Le nouveau roman. Une césure dans l'histoire du récit, Paris, Nathan. 2001.

5- JOPECK (Sylvie): La photographie et l'autobiographie, anthologie, Paris, Editions Gallimard, 2004

6- PAQUIN (Michel): Lecture de roman, La lignée, 1984 
7- PRECKSHOT (Judith): L'analyse du récit: lexique commenté, à travers l'internet.

8- REUTER (Yves): Introduction à l'analyse du roman, Bordas, Paris, 1991

9- ROBBE-GRILLET (Alain): Pour un nouveau roman, Paris, Gallimard, 1963

10-ROHOU (Jean): Les études littératures "guide de l'étudiant", Nathan, Tours, 1993

11-ROMERAL (Francesca): Les Années 80 et l'épanouissement de l'autofiction, Intercambio, $2^{\text {ème }}$ série, vol. 6, 2013

12-VERCIER (Bruno): La littérature en France depuis 1968, Bordas, 1982, P.P. 240

III- Références consultées en français:

1- BARTHES (Roland): Critique et vérité, Edition du Seuil VI, 1966.

2- BARTHES (Roland): Essais critiques, Edition du Seuil VI, 1964.

3- BOISDEFFRE (Pierre) : Où va le roman? Paris, Editions Mondiales, 1972.

4- GENETTE (Gérard) : Figures III, Paris VI, Edition du Seuil, 1972.

5- GOLDENSTIEN (Jean Pierre) : Lire le roman, Bruxelles, De Boeck Duculot, 1999.

6- GOLDMAN (Lucien): Pour une sociologie du roman, Paris, Gallimard, 1964.

7- MITTERAND (Henri): Le discours du roman, Presses Universitaires de France, 1986.

8- PATILLON (Michel): Précis d'analyse littéraire, Nathan, 1986.

9- RULLIER-THEURET (François) : Approche du roman, Hachette, 2001.
10-THUMEREL (Fabrice): La critique littéraire, Armand colin, 1998.

IV- Références en arabe:

$$
\text { 1- رشيد (امينة) : ترجمة رواية المكان, دار }
$$

V-Thèses et recherches :

1- Hugueny (Elise): Transgressions textuelles, déplacements littéraires et enjeux sociopolitique du transpersonnel dans l'œuvre d'Annie Ernaux, thèse de doctorat, université de Durham, Angleterre, 2007

2- Soron (Antony): La Place d'Annie ou le délit d'appartenance normande, Sorbonne, Paris.

VI- Périodiques:

1- Entretien avec Jean Royer, Le Devoir, 26 mars 1988

2- Ernaux (Annie) "écrire, pourquoi?" Entretien avec Raphaëlle Rerolle, Editions de la bibliothèque publique d'information- Centre Pompidou, 2010.

3- Ghafouri (Layla): La Revue de Téhéran, mensuel culturel iranien en langue française, n. 94, septembre, 2013.

4- L'écriture comme un couteau, entretien avec Fréderic, Yves Jeannet, Paris, Stock, 2003

\section{VII- Médias:}

-INA. Fr., Archive INA, Annie Ernaux, La place, You tube

\section{VIII-SITOGRAPHIE :}

1- http://www.dissertations gratuites. Com/dissertations/pour-Analyserun-roman $/ 1322 \mathrm{html}$ http// www érudit. Org. /à propos/ utilisation html 